\title{
Early intervention in psychosis: keeping the body in mind
}

Most GPs can remember being faced with a young person experiencing their first psychotic illness: the patient's confusion, their fear, their family's distress. Perhaps some will recall ambivalence about invoking the Mental Health Act, or contacting colleagues to cover surgery while awaiting the arrival of another doctor or the police. The shared sense of bewilderment and sadness may have been balanced by some personal relief that normal working life might soon resume after the hours, days, or maybe weeks of distress, phone calls, and visits.

Psychosis affects young people. More than three-quarters of men and two-thirds of women who experience psychosis have their first episode by age 35 years; most are in their late teens and twenties. ${ }^{1}$ This has prompted the development of more 'youth sensitive' early intervention services in England and, to a lesser extent, in Scotland and Wales. A 3-year package of intensive, specialist, and age-appropriate mental health care results in less traumatic engagement and improved follow-up., ${ }^{2,3}$

Lester et al, in the current issue of the $B J G P$, researched whether an educational intervention to raise GPs' awareness of presenting clinical features of first episode psychosis could encourage earlier referral. ${ }^{4}$ While few would deny the importance of improving these care pathways, the study suggests that promoting diagnostic awareness adds little by itself: GPs are already quite good at detection. What Lester et al did find was that training could facilitate collaboration between GPs and the new early intervention services, and the obstacles were more about how they worked together. Moreover, evidence is now emerging about future physical health problems for these young people. This opens an important new theme which might benefit from strengthened collaboration between specialist early intervention services and primary care, utilising the latter's expertise in cardiovascular risk management.

People with a psychotic illness (schizophrenia and bipolar disorder) die up to 25 years earlier than the general population. ${ }^{5}$ While suicide or injury causes about one-third of these premature deaths, two-thirds result from physical health's 'usual suspects': cardiovascular, pulmonary, and infectious diseases. ${ }^{6}$ The Disability Rights Commission (now the Equality and Human Rights Commission) highlighted how people with severe mental illness experience extreme health inequalities. ${ }^{7}$ Despite continuing government policy to narrow health inequalities and improve primary prevention of cardiovascular disease in the general population, this differential mortality gap is actually worsening, particularly from heart disease in younger people where, for instance, those aged 25 to 44 years with psychosis are 6.6 times more likely to die prematurely than the general population. ${ }^{8}$

Like most health issues, a variety of factors are implicated. The general population in developed countries, like the UK, is experiencing an escalation in cardiovascular risk factors, such as obesity and less exercise, and more type 2 diabetes mellitus. Superimposed on that are lifestyle issues (not all actively chosen) for people with psychosis, generating a potent and interactive escalation of cardiovascular risks. ${ }^{9}$ In particular, people with psychosis may lead more sedentary lives, eat less fruit and vegetables, be much more likely to be obese, are two to three times more likely to smoke cigarettes, and five times more likely to smoke heavily. ${ }^{10}$ Greater cannabis use provides a 'double whammy', stimulating appetite and likelihood of obesity, as well as being a 'pro-psychotic' drug for some. ${ }^{11}$ People with psychosis face a future of social exclusion and poverty, where only $24 \%$ have employment, one in three are in debt, and one in four have serious rent arrears. ${ }^{12}$

Tobacco consumption is particularly important given that $64 \%$ of those with probable psychosis smoke compared with $29 \%$ without psychosis in a large population survey of psychiatric morbidity. ${ }^{13}$ Moreover, $76 \%$ of people with a first episode of psychosis are regular smokers. ${ }^{14}$ Smoking is the largest UK contributor to preventable illness and explains much of the excess mortality of people with mental health problems. ${ }^{6}$ Moreover, smokers experience $44 \%$ more type 2 diabetes compared with non-smokers, rising to $61 \%$ for those smoking over 20 cigarettes a day, ${ }^{15}$ providing a potent interaction between risks which link to mental disorder.

Another major concern is the adverse impact of antipsychotic medicines on cardiovascular risk. Widespread adoption of so-called second generation or atypical antipsychotic medicines over the past 15 years took place in the hope that they would be more effective than older, firstgeneration compounds, partly because a reduced side-effect burden would enhance treatment adherence. However, recent evidence indicates this hope was misplaced..$^{16}$ There are few, if any, class differences in effectiveness and while neurological side-effects may be less prominent with newer antipsychotics, attention has shifted to adverse effects on weight, glucose metabolism, and lipid profile. ${ }^{17}$ Any antipsychotic drug, clozapine and olanzapine in particular, can worsen cardiovascular risk profile. Furthermore, specific genetic factors also operate: $15 \%$ of drug-naïve individuals with first episode psychosis already have elevated fasting glucose levels, and high levels of insulin and cortisol, accompanied by three times as much intra-abdominal fat as matched controls. ${ }^{18}$ Therefore, clinicians should avoid arbitrary classification of antipsychotic drugs and should tailor treatment to maximise benefits and minimise sideeffects, including metabolic abnormalities, in individual patients.

Finally, there is some suggestion of discrimination by health professionals; for example, screening rates for diabetes in routine clinical practice in psychiatry and primary care appear sub-optimal, ${ }^{19}$ and people with comorbid psychosis and diabetes may receive fewer routine eye checks and have poorer glycaemic and lipid control. ${ }^{20}$ Despite higher general practice consultation rates (13-14 consultations per 
year for people with psychosis compared with approximately three per year for the general population), data recording for a range of health promotion areas was significantly less likely. Cardiovascular risk factors, in particular, were less likely to be recorded or acted upon. ${ }^{2}$

The evidence base and implementation mechanisms to improve physical healthcare of people with first episode psychosis are in place. The Quality and Outcomes Framework has incentivised annual physical health checks for people with psychosis since 2004, now also recommended by the new schizophrenia guidelines from the National Institute for Health and Clinical Excellence. ${ }^{22}$ Furthermore, there is some evidence of mental health benefits from healthy living interventions on smoking cessation, weight management, and exercise. ${ }^{23}$ What is perhaps lacking is a more general recognition that primary care can define a group of many thousands of young people in their twenties and thirties, at ages primary care would not normally consider for active primary or secondary cardiovascular prevention, who are at high risk of dying young.

GPs are often more comfortable discussing physical than mental health issues with patients with psychosis, seeing that as their core role. ${ }^{24}$ Primary care and specialist mental health clinicians could work together to provide clear and consistent information to help patients understand and weigh the benefits and risks of antipsychotic medication, emphasising the trade-offs of improved mental health symptoms versus increased risks to physical health. This is similar to the way in which cancer specialists and GPs routinely discuss the secondary morbidity of potentially curative treatments, and no less important. Perhaps we could target these young people proactively to help them understand their physical health risks, encourage their participation in care decisions, and engage in positive actions to improve physical wellbeing.

Above all, a different mindset about early intervention in psychosis is needed in primary care. It is worth reflecting that if these young people had diabetes and not psychosis, would we strive more energetically to reduce cardiovascular risk?

A diagnosis of psychosis is as much a marker of cardiovascular risk as diabetes, and should invoke a similar intensity of concern, investigation, and management.

Like diabetes, most morbidity occurs in the larger group at medium risk as there are adverse effects on glycosylated haemoglobin regardless of whether the actual figure crosses a recognised audit threshold. A key message here is that all the risk parameters - lipids, glycaemic control, smoking, blood pressure, and body mass index - are adversely affected by a diagnosis of and treatment for psychosis. Discussions over therapeutic decisions and screening and intervention for cardiovascular risk factors should commence as a standard, right from the start, for every young person with psychosis.

The paradigm of early intervention for this high risk population should encompass mind and body. Primary care, with its strengths of holism and its skills and experience of providing preventive care, is well placed to understand and implement such approaches. Educational programmes that promote early intervention in psychosis, as highlighted by Lester et $\mathrm{al},{ }^{4}$ may need to broaden their focus from simply early-stage detection to encompass these wider physical health aspects.

\section{David Shiers,}

GP and Joint Lead to National Early Intervention in Psychosis Programme, National Mental Health Development Unit, London.

\section{Peter B Jones,}

Hon Consultant Psychiatrist, CAMEO Early Intervention Services, Cambridgeshire and Peterborough Foundation Trust.

\section{Steve Field,}

GP, Bellevue Medical Centre, Birmingham; Chairman of the RCGP.

\section{Provenance}

Commissioned; peer reviewed.

\section{REFERENCES}

1. Kirkbride JB, Fearon P, Morgan C, et al. Heterogeneity in incidence rates of schizophrenia and other psychotic syndromes: findings from the 3-center AeSOP study. Arch Gen Psychiatry 2006; 63(3): 250-258.

2. Craig T, Garety P, Power P, et al. The Lambeth Early Onset (LEO) team: randomised controlled trial of the effectiveness of specialised care for early psychosis. BMJ 2004; 329: 1067-1070.

3. Nordentoft M, Jeppesen P, Kassow P, et al. OPUS project: a randomized controlled trial of integrated psychiatric treatment in first episode psychosis - clinical outcome improved. Schizophr Res 2002; 53(Suppl 1): 51.

4. Lester H, Birchwood M, Freemantle N, et al. REDIRECT: cluster randomised controlled trial of GP training in first-episode psychosis. Br J Gen Pract 2009; 59: 403-408.

5. Parks J, Svendsen D, Singer P, Foti ME (eds). Morbidity and mortality in people with serious mental illness. Alexandria, Virginia: National Association of State Mental Health Program Directors (NASMHPD) Medical Directors Council, 2006.

6. Brown S. Excess mortality of schizophrenia. A metaanalysis. Br J Psychiatr 1997; 171: 502-508.

7. Disability Rights Commission. Equal treatment: closing the gap. A formal investigation into physical health inequalities experienced by people with learning disabilitie and/or mental health problems. London: DRC, 2005.

8. Saha S, Chant D, McGrath J. A systematic review of mortality in schizophrenia: is the differential mortality gap worsening over time? Arch Gen Psychiatry 2007; 64(10): 1123-1131.

9. Heiskanen T, Niskanen L, Lyytikäinen R, et al. Metabolic syndrome in patients with schizophrenia.
J Clin Psychiatry 2003; 64(5): 575-579.

10. McCreadie R, MacDonald E, Blacklock C. et al. Dietary intake of schizophrenic patients in Nithsdale, Scotland: case-control study. BMJ 1998; 317(7161): 784-785.

11. Fergusson DM, Poulton R, Smith PF, Boden JM. Cannabis and psychosis. BMJ 2006; 332(7534): 172-175.

12. Office of Deputy Prime Minister. Mental health and social exclusion: Social Exclusion Unit report. London: Office of Deputy Prime Minister, 2004; 11-12.

13. Coulthard M, Farrell M, Singleton N, Meltzer H. Tobacco, alcohol and drug use and mental health. London: Stationery Office, 2002.

14. Wade D, Harrigan S, Edwards J, et al. Course of substance misuse and daily tobacco use in first-episode psychosis. Schizophrenia Res 2006; 81(2-3): 145-150.

15. Willi C, Bodenmann P, Ghali WA et al. Active smoking and the risk of type 2 diabetes: a systematic review and meta-analysis. JAMA 2007; 298(22): 2654-2664.

16. Tyrer P, Kendall T. The spurious advance of antipsychotic drug therapy. Lancet 2009; 373(9657): 4-5

17. Newcomer JW. Second-generation (atypical) antipsychotics and metabolic effects: a comprehensive literature review. CNS Drugs 2005; 19(Suppl 1): 1-93.

18. Ryan MC, Collins $P$, Thakore JH. Impaired fasting glucose tolerance in first-episode, drug-naive patients with schizophrenia. Am J Psychiatr 2003; 160(2): 284-289.

19. Tarrant CJ. Blood glucose testing for adults prescribed atypical antipsychotics in primary and secondary care. Psychiatric Bulletin 2006; 30(8): 286-288.

20. Frayne SM, Halanych JH, Miller DR et al. Disparities in diabetes care: impact of mental illness. Arch Intern Med 2005; 165(22): 2631-2638.

21. Kendrick T. Cardiovascular and respiratory risk factors and symptoms among general practice patients with long-term mental illness. Br J Psychiatry 1996; 169(6): 733-739.

22. National Institute for Health and Clinical Excellence. $C G$ 82 Schizophrenia: core interventions in the treatment and management of schizophrenia in primary and secondary care (update). Clinical Guideline. London: NICE, 2009.

23. Bradshaw T, Lovell K, Harris N. Healthy living interventions and schizophrenia: a systematic review. JAdv Nurs 2005; 49(6): 634-654.

24. Lester H, Tritter JQ, Sorohan H. Patients' and health professionals' views on primary care for people with serious mental illness: focus group study. BMJ 2005; 330(7500): 1122

DOI: 10.3399/bjgp09X420888

\section{ADDRESS FOR CORRESPONDENCE}

\section{David Shiers}

National Mental Health Development Unit Department of Health,

Area 306, Wellington House,

133-155 Waterloo Road, London.

E-mail: david.shiers@nmhdu.org.uk 